\title{
Class Scheme Class Scheme Item
}

National Cancer Institute

\section{Source}

National Cancer Institute. Class Scheme Class Scheme Item. NCI Thesaurus. Code C41081.

This builds the relationships between Classification Schemes and Classification Scheme Items. 